\title{
Quasilinearization for fractional differential equations of Riemann-Liouville type
}

\author{
Zhenhai Liu, Rui Wang, and Jing Zhao
}




\title{
QUASILINEARIZATION FOR FRACTIONAL DIFFERENTIAL EQUATIONS OF RIEMANN-LIOUVILLE TYPE
}

\author{
ZHENHAI LIU, RUI WANG, AND JING ZHAO
}

Received 11 October, 2013

\begin{abstract}
In this paper, we deal with the quasilinearization for Riemann-Liouville fractional differential equations with two point boundary condition. By establishing a new comparison principle we get a monotone sequence which converges quadratically to the unique solution of the fractional differential equations.
\end{abstract}

2010 Mathematics Subject Classification: 26A33; 93C15

Keywords: quasilinearization, fractional differential equations, monotone sequence, quadratic convergence

\section{INTRODUCTION}

Recently, the theory of fractional differential equations become a hot topic in many fields. It is known that many physical system can be represented more accurately through fractional derivative formulation. There are many fields of applications where we can use the fractional calculus as the mathematical model of systems and processes in the fields of physics, chemistry, aerodynamics, electrodynamics of complex medium, viscoelasticity, heat conduction, electricity mechanics, control theory, and so forth. For more details on the topics one can see for instance, $[7-9,11-13,15,16,20]$ and the reference therein..

Many authors have studied fractional differential equations from two aspects, one is the theoretical aspects of the existence and uniqueness of solutions [1, 5, 6, 22, 23], the other is the development of analytic and numerical methods for finding solutions. The numerical-analytical technique based on successive approximations leads us the approximate solutions to differential equations. Now, it has been studied widely in the theory of integer differential equations, see for instance [17-19].

Project supported by NNSF of China Grants Nos.11271087, 61263006, Scientific Research Project of Guangxi EducationDepartment,China (no. 2013YB074), Scientific Research Project of State Ethnic Affairs Commission of China (no. 12GXZ001). 
It is well known that the method of quasilinearization [10] offers an approach for obtaining approximate solutions to differential equations. This method not only applies to integer differential equations, but also applies to the fractional differential equations, which combining with the lower and upper solution method enables us to get a monotone sequence which converge quadratically to the solution of the differential equations(cf. [2-4, 14, 21]).

In $[2,4]$, the authors studied the following Caputo fractional differential equations by using the quasilinearization method:

$$
\left\{\begin{array}{l}
{ }^{c} D_{0+}^{q} x(t)=f(t, x(t)), t \in J=\left[t_{0}, T\right], \\
x\left(t_{0}\right)=x_{0},
\end{array}\right.
$$

where $0<q \leq 1, f \in C(J \times R, R)$.

Later on, in [3], Vasundhara Devi and Radhika developed the quasilinearization method for the Caputo fractional impulsive differential systems as:

$$
\left\{\begin{array}{l}
\left.{ }^{c} D_{0+}^{q} x(t)=f(t, x(t))\right), t \neq t_{k}, \\
x\left(t_{k}^{+}\right)=I_{k}\left(x\left(t_{k}\right)\right), k=1,2, \cdots n-1, \\
x\left(t_{0}\right)=x_{0},
\end{array}\right.
$$

where $0<q \leq 1, f \in P C\left(\left[t_{0}, T\right] \times R, R\right), I_{k}: R \rightarrow R$. The comparison theorem they used is developed from differential equation.

Motivated by the works mentioned above, we investigate the following nonlinear two point boundary value problem for Riemann-Liouville fractional differential equations:

$$
\left\{\begin{array}{l}
D_{0+}^{q} x(t)=f(t, x(t)), t \in(0, T], \\
\tilde{x}(0)=g(\tilde{x}(T)),
\end{array}\right.
$$

where $J \in[0, T], \frac{1}{2}<q \leq 1, f \in C(J \times R, R), g \in C(R, R)$ and $\tilde{x}(0)=\left.t^{1-q} x(t)\right|_{t=0}, \tilde{x}(T)=\left.t^{1-q} x(t)\right|_{t=T}$. We develop a new comparison principle and the method of quasilinearization for Riemann-Liouville fractional differential equations.

Significant progresses have been made to the quasilinearization of the Caputo fractional differential equations (cf.[2-4]). However, to our best knowledge, the quasilinearization for Riemann-Liouville fractional differential equations is still an untreated topics in the literature and this fact is the motivation of the present work. Our aim in this paper is to provide some suitable sufficient conditions for the existence and uniqueness of solutions and approximate results for Riemann-Liouville fractional differential equations.

This paper is organized as follows. In Section 2, we provide some definitions, lemmas and establish a new comparison principle by integral equations. In Section 3 , The lower and upper solutions method and quasilinearization method are used to 
construct a monotone sequence which converge uniformly and quadratically to the unique solution of (1.1).

\section{PReliminaries}

In this section, we introduce some notations, definitions and preliminary facts which are used throughout this paper.

Let $C_{1-\alpha}(J, R)=\left\{x \in C(0, T]: t^{1-\alpha} x(t) \in C[0, T]\right\}$ with the norm $\|x\|_{C_{1-\alpha}}=$ $\max _{t \in J}\left|t^{1-\alpha} x(t)\right|$. Obviously, the space $C_{1-\alpha}(J, R)$ is a Banach space. The following definitions can be found from $[8,16]$ :

Definition 1. The integral

$$
I_{0+}^{\alpha} f(t)=\frac{1}{\Gamma(\alpha)} \int_{0}^{t} \frac{f(s)}{(t-s)^{1-\alpha}} d t, \quad \alpha>0,
$$

is called Riemann-Liouville fractional integral of order $\alpha$, where $\Gamma$ is the gamma function.

Definition 2. For a function $f(t)$, the Riemann-Liouville derivative of order $\alpha$ can be written as

$$
D_{0+}^{\alpha} f(t)=\left(\frac{d}{d t}\right)^{n}\left(I_{0+}^{n-\alpha} f(t)\right)=\frac{1}{\Gamma(n-\alpha)} \frac{d^{n}}{d t^{n}} \int_{0}^{t}(t-s)^{n-\alpha-1} f(s) d s,
$$

where $n-1<\alpha \leq n$.

Lemma 1 ([8]). Let $n-1<\alpha \leq n$ and let $f_{n-\alpha}(t)=I_{0+}^{n-\alpha} f(t)$ be the fractional integral of order $n-\alpha$. If $f(t) \in L(0, T)$ and $f_{n-\alpha}(t) \in A C^{n}[0, T]$, then we have the following equality

$$
I_{0+}^{\alpha} D_{0+}^{\alpha} f(t)=f(t)-\sum_{i=1}^{n} \frac{f_{n-\alpha}^{(n-i)}(0)}{\Gamma(\alpha-i+1)} t^{\alpha-i} .
$$

Lemma 2. Let $\sigma \in C_{1-q}(J, R) . x \in C_{1-q}(J, R)$ is a solution of the following linear initial value problem:

$$
\left\{\begin{array}{l}
D_{0+}^{q} x(t)=M x(t)+\sigma(t), \quad t \in(0, T], 0<q \leq 1, \\
\tilde{x}(0)=N \tilde{x}(T)+r, \quad r \in R,
\end{array}\right.
$$

if and only if $x(t)$ is a solution of the following integral equation:

$$
x(t)=(N \tilde{x}(T)+r) t^{q-1}+\frac{1}{\Gamma(q)} \int_{0}^{t}(t-s)^{q-1}(\sigma(s)+M x(s)) d s,
$$

where $M, N$ are constants. 
Proof. Assume $x(t)$ satisfies (2.1). From the first equation of (2.1) and Lemma 1, we have

$$
\begin{aligned}
x(t) & =\frac{\left.I_{0+}^{1-q} x(t)\right|_{t=0} t^{q-1}}{\Gamma(q)}+I_{0+}^{q}(\sigma(t)+M x(t)) \\
& =(N \tilde{x}(T)+r) t^{q-1}+\frac{1}{\Gamma(q)} \int_{0}^{t}(t-s)^{q-1}(\sigma(s)+M x(s)) d s .
\end{aligned}
$$

Conversely, assume that $x(t)$ satisfies (2.2). It is easy to check that $x(t) \in C_{1-q}(J, R)$. Applying the operator $D_{0+}^{q}$ to both sides of (2.2), we have

$$
D_{0+}^{q} x(t)=M x(t)+\sigma(t) .
$$

In addition, by simple calculation, we conclude $\tilde{x}(0)=\left.t^{1-q} x(t)\right|_{t=0}=N \tilde{x}(T)+$ $r$.

Lemma 3. Assume that $M, N \geq 0$ are constants and the following inequality holds

$$
N+\frac{M T^{q} \Gamma(q)}{\Gamma(2 q)}<1
$$

then (1.1) has a unique solution.

Proof. We firstly define an operator $F: C_{1-q}(J, R) \rightarrow C_{1-q}(J, R)$ by

$$
(F x)(t)=(N \tilde{x}(T)+r) t^{q-1}+\frac{1}{\Gamma(q)} \int_{0}^{t}(t-s)^{q-1}(\sigma(s)+M x(s)) d s .
$$

It is easy to check that $t^{1-q}(F x)(t) \in C(J, R)$. Hence the operator $F$ is well defined on $C_{1-q}(J, R)$.

For any $x, y \in C_{1-q}(J, R)$, we have

$$
\begin{aligned}
& \|F x-F y\|_{C_{1-q}} \\
& =\max _{t \in J}\left|t^{1-q}[(F x)(t)-(F y)(t)]\right| \\
& \leq \max _{t \in J}|N(\tilde{x}(T)-\tilde{y}(T))|+\max _{t \in J} \frac{t^{1-q}}{\Gamma(q)} \int_{0}^{t}(t-s)^{q-1} M s^{q-1} s^{1-q}|x(s)-y(s)| d s \\
& \leq N\|x-y\|_{C_{1-q}}+\max _{t \in J} \frac{M t^{1-q}}{\Gamma(q)} \int_{0}^{t}(t-s)^{q-1} s^{q-1} d s\|x-y\|_{C_{1-q}} \\
& \leq\left(N+\frac{M T^{q} \Gamma(q)}{\Gamma(2 q)}\right)\|x-y\|_{C_{1-q}} .
\end{aligned}
$$

According to (2.3) and the Banach fixed point theorem, (1.1) has a unique solution. 
Definition 3. A function $\alpha_{0} \in C_{1-q}(J, R)$ is called a lower

$$
\left\{\begin{array}{l}
D_{0+}^{q} \alpha_{0} \leq \tilde{\alpha_{0}}(0) t^{q-1}+\frac{1}{\Gamma(q)} \int_{0}^{t}(t-s)^{q-1} f\left(s, \alpha_{0}(s)\right) d s, \\
\tilde{\alpha_{0}}(0) \leq g\left(\tilde{\alpha_{0}}(T)\right) .
\end{array}\right.
$$

Analogously, $\beta_{0} \in C_{1-q}(J, R)$ is called an upper solution of (1.1) if

$$
\left\{\begin{array}{l}
D_{0+}^{q} \beta_{0} \geq \tilde{\beta_{0}}(0) t^{q-1}+\frac{1}{\Gamma(q)} \int_{0}^{t}(t-s)^{q-1} f\left(s, \beta_{0}(s)\right) d s, \\
\tilde{\beta_{0}}(0) \geq g\left(\tilde{\beta_{0}}(T)\right) .
\end{array}\right.
$$

In what follows, we assume that

$$
\alpha_{0}(t) \leq \beta_{0}(t), t \in(0, T], \tilde{\alpha_{0}}(0) \leq \tilde{\beta_{0}}(0) .
$$

Let $\left[\alpha_{0}, \beta_{0}\right]=\left\{x \in C_{1-q}(J, R): \alpha_{0}(t) \leq x(t) \leq \beta_{0}(t), \forall t \in(0, T], \tilde{\alpha_{0}}(0) \leq \tilde{x_{0}}(0) \leq\right.$ $\left.\tilde{\beta_{0}}(0)\right\}$.

Lemma 4. Suppose that there are two constants $M, N \geq 0$ such that

$$
0 \leq f_{x}(t, \eta(t)) \leq M, 0 \leq g^{\prime}(\tilde{\eta}(T)) \leq N, \forall \eta \in\left[\alpha_{0}, \beta_{0}\right]
$$

If (2.3) holds and $p(t) \in C_{1-q}(J, R)$ satisfies

$$
\left\{\begin{array}{l}
p(t) \leq \tilde{p}(0) t^{q-1}+\frac{1}{\Gamma(q)} \int_{0}^{t}(t-s)^{q-1} f_{x}(s, \eta(s)) p(s) d s, \\
\tilde{p}(0) \leq g^{\prime}(\tilde{\eta}(T)) \tilde{P}(T),
\end{array}\right.
$$

then $p(t) \leq 0$ for all $t \in(0, T]$ and $\tilde{p}(0) \leq 0$.

Proof. Suppose that the inequality $p(t) \leq 0, \forall t \in(0, T]$ is not true. So there exists at least a $t_{*} \in(0, T]$ such that $t_{*}^{1-q} p\left(t_{*}\right)>0$. Without loss of generality, we assume that $t_{*}^{1-q} p\left(t_{*}\right)=\max \left\{t^{1-q} p(t): t \in(0, T]\right\}=\rho>0$.

Then we have that

$$
\begin{aligned}
t^{1-q} p(t) & \leq \tilde{p}(0)+\frac{t^{1-q}}{\Gamma(q)} \int_{0}^{t}(t-s)^{q-1} f_{x}(s, \eta(s)) p(s) d s \\
& \leq g^{\prime}(\tilde{\eta}(T)) \tilde{P}(T)+\frac{t^{1-q}}{\Gamma(q)} \int_{0}^{t}(t-s)^{q-1} f_{x}(s, \eta(s)) p(s) d s \\
& \leq N \rho+\frac{t^{1-q}}{\Gamma(q)} \int_{0}^{t}(t-s)^{q-1} s^{q-1} f_{x}(s, \eta(s)) s^{1-q} p(s) d s .
\end{aligned}
$$

Let $t=t_{*}$, we have

$$
\rho \leq\left(N+\frac{M T^{q} \Gamma(q)}{\Gamma(2 q)}\right) \rho .
$$

So

$$
N+\frac{M T^{q} \Gamma(q)}{\Gamma(2 q)} \geq 1
$$


Which contradicts (2.3). Hence $p(t) \leq 0$ for all $t \in(0, T]$.

For $t=0, \tilde{p}(T) \leq 0$, we have that

$$
\tilde{p}(0) \leq g^{\prime}(\tilde{\eta}(T)) \tilde{P}(T) \leq 0 .
$$

\section{QUASILINEARIZATION}

In this section, we use the method of quasilinearization to construct a monotone sequence which converge quadratically to the solution of fractional differential equations with two point boundary condition.

Theorem 1. Let $\alpha_{0}, \beta_{0}$ be a lower and upper solutions of (1.1), respectively. Assume that (2.3) holds and that

(1) $0 \leq g^{\prime}(\tilde{\eta}(T)) \leq N, 0 \leq g^{\prime}(x)-g^{\prime}(y) \leq L_{1}(x-y)$,

(2) $0 \leq f_{x}(t, \eta(t)) \leq M, 0 \leq f_{x}(t, x)-f_{x}(t, y) \leq L_{2}(x-y)$, where $L_{1}, L_{2} \geq 0$, $\eta \in\left[\alpha_{0}, \beta_{0}\right]$ and $\alpha_{0} \leq y \leq x \leq \beta_{0}$,

(3) $1-\Gamma(q) E_{q, q}\left(M T^{q}\right) N>0$.

Then there exist two monotone sequences $\left\{\alpha_{n}\right\},\left\{\beta_{n}\right\} \subset\left[\alpha_{0}, \beta_{0}\right]$ both of which converge uniformly to the unique solution of (1.1) and the convergence is quadratic.

Proof. For any $\eta \in\left[\alpha_{0}, \beta_{0}\right]$, consider the following linear fractional differential equation:

$$
\left\{\begin{array}{l}
D_{0+}^{q} x(t)=f(t, \eta(t))+f_{x}(t, \eta(t))(x(t)-\eta(t)), \\
\tilde{x}(0)=g(\tilde{\eta}(T))+g^{\prime}(\tilde{\eta}(T))(\tilde{x}(T)-\tilde{\eta}(T)) .
\end{array}\right.
$$

Obviously, by Lemma 3, the problem above has a unique solution which satisfies $\left\{\begin{array}{l}x(t)=\tilde{x}(0) t^{q-1}+\frac{1}{\Gamma(q)} \int_{0}^{t}(t-s)^{q-1}\left[f(s, \eta(s))+f_{x}(s, \eta(s))(x(s)-\eta(s))\right] d s, \\ \tilde{x}(0)=g(\tilde{\eta}(T))+g^{\prime}(\tilde{\eta}(T))(\tilde{x}(T)-\tilde{\eta}(T)) .\end{array}\right.$

Replacing $\eta, x$ by $\alpha_{0}, \alpha_{1}$, respectively, we obtain

$$
\left\{\begin{aligned}
\alpha_{1}(t) & =\tilde{\alpha_{1}}(0) t^{q-1} \\
& +\frac{1}{\Gamma(q)} \int_{0}^{t}(t-s)^{q-1}\left[f\left(s, \alpha_{0}(s)\right)+\tilde{f_{x}}\left(s, \alpha_{0}(s)\right)\left(\alpha_{1}(s)-\alpha_{0}(s)\right)\right] d s, \\
\tilde{\alpha_{1}}(0) & =g\left(\tilde{\alpha_{0}}(T)\right)+g^{\prime}\left(\tilde{\alpha_{0}}(T)\right)\left(\tilde{\alpha_{1}}(T)-\tilde{\alpha_{0}}(T)\right) .
\end{aligned}\right.
$$

Setting $p(t)=\alpha_{0}(t)-\alpha_{1}(t)$, we obtain that

$$
\begin{aligned}
p(t) & =\alpha_{0}(t)-\alpha_{1}(t) \\
& \leq \tilde{\alpha_{0}}(0) t^{q-1}+\frac{1}{\Gamma(q)} \int_{0}^{t}(t-s)^{q-1} f\left(s, \alpha_{0}(s)\right) d s-\tilde{\alpha_{1}}(0) t^{q-1} \\
& -\frac{1}{\Gamma(q)} \int_{0}^{t}(t-s)^{q-1}\left[f\left(s, \alpha_{0}(s)\right)+f_{x}\left(s, \alpha_{0}(s)\right)\left(\alpha_{1}(s)-\alpha_{0}(s)\right)\right] d s
\end{aligned}
$$




$$
\leq \tilde{p}(0) t^{q-1}+\frac{1}{\Gamma(q)} \int_{0}^{t}(t-s)^{q-1} f_{x}\left(s, \alpha_{0}(s)\right) p(s) d s .
$$

And we have that

$$
\begin{aligned}
\tilde{p}(0) & =\tilde{\alpha_{0}}(0)-\tilde{\alpha_{1}}(0) \\
& \leq g\left(\tilde{\alpha_{0}}(T)\right)-g\left(\tilde{\alpha_{0}}(T)\right)-g^{\prime}\left(\tilde{\alpha_{0}}(T)\right)\left(\tilde{\alpha_{1}}(T)-\tilde{\alpha_{0}}(T)\right) \\
& =g^{\prime}\left(\tilde{\alpha_{0}}(T)\right) \tilde{p}(T) .
\end{aligned}
$$

By Lemma 4, we know $p(t) \leq 0$ for all $t \in(0, T]$ and $\tilde{p}(0) \leq 0$. So $t^{1-q} \alpha_{0}(t) \leq$ $t^{1-q} \alpha_{1}(t)$ for all $t \in J$.

Now replacing $\eta, x$ by $\beta_{0}, \beta_{1}$, we have that

$$
\left\{\begin{aligned}
\beta_{1}(t)= & \tilde{\beta}_{1}(0) t^{q-1} \\
& +\frac{1}{\Gamma(q)} \int_{0}^{t}(t-s)^{q-1}\left[f\left(s, \beta_{0}(s)\right)+f_{x}\left(s, \alpha_{0}(s)\right)\left(\beta_{1}(s)-\beta_{0}(s)\right)\right] d s, \\
\tilde{\beta_{1}}(0)= & g\left(\tilde{\beta_{0}}(T)\right)+g^{\prime}\left(\tilde{\alpha_{0}}(T)\right)\left(\tilde{\beta_{1}}(T)-\tilde{\beta_{0}}(T)\right) .
\end{aligned}\right.
$$

Similarly, we can get $t^{1-q} \beta_{1}(t) \leq t^{1-q} \beta_{0}(t)$ for all $t \in J$.

Next we set $p(t)=\alpha_{1}(t)-\beta_{1}(t)$, we can obtain

$$
\begin{gathered}
p(t)=\alpha_{1}(t)-\beta_{1}(t) \\
=\tilde{\alpha_{1}}(0) t^{q-1}+\frac{1}{\Gamma(q)} \int_{0}^{t}(t-s)^{q-1}\left[f\left(s, \alpha_{0}(s)\right)+f_{x}\left(s, \alpha_{0}(s)\right)\left(\alpha_{1}(s)-\alpha_{0}(s)\right) d s\right] \\
-\tilde{\beta_{1}(0) t^{q-1}}-\frac{1}{\Gamma(q)} \int_{0}^{t}(t-s)^{q-1}\left[f\left(s, \beta_{0}(s)\right)+f_{x}\left(s, \alpha_{0}(s)\right)\left(\beta_{1}(s)-\beta_{0}(s)\right)\right] d s \\
\leq \tilde{p}(0) t^{q-1}+\frac{1}{\Gamma(q)} \int_{0}^{t}(t-s)^{q-1} f_{x}\left(s, \alpha_{0}(s)\right) p(s) d s .
\end{gathered}
$$

And we have that

$$
\begin{aligned}
\tilde{p}(0) & =\tilde{\alpha_{1}}(0)-\tilde{\beta_{1}}(0) \\
& =g\left(\tilde{\alpha_{0}}(T)\right)-g\left(\tilde{\beta_{0}}(T)\right)+g^{\prime}\left(\tilde{\alpha_{0}}(T)\right)\left[\tilde{\alpha_{1}}(T)-\tilde{\alpha_{0}}(T)-\tilde{\beta_{1}}(T)+\tilde{\beta_{0}}(T)\right] \\
& \leq g^{\prime}\left(\tilde{\alpha_{0}}(T)\right) \tilde{p}(T) .
\end{aligned}
$$

By Lemma 4, we know $p(t) \leq 0$ for all $t \in(0, T]$ and $\tilde{p}(0) \leq 0$. So $t^{1-q} \alpha_{1}(t) \leq$ $t^{1-q} \beta_{1}(t)$ for all $t \in J$.

Hence, we have $t^{1-q} \alpha_{0}(t) \leq t^{1-q} \alpha_{1}(t) \leq t^{1-q} \beta_{1}(t) \leq t^{1-q} \beta_{0}(t)$ for all $t \in J$.

Now suppose that

$$
t^{1-q} \alpha_{0}(t) \leq t^{1-q} \alpha_{k-1}(t) \leq t^{1-q} \alpha_{k}(t) \leq t^{1-q} \beta_{k}(t) \leq t^{1-q} \beta_{k-1}(t) \leq t^{1-q} \beta_{0}(t) .
$$


To show $t^{1-q} \alpha_{k}(t) \leq t^{1-q} \alpha_{k+1}(t) \leq t^{1-q} \beta_{k+1}(t) \leq t^{1-q} \beta_{k}(t)$.

It's easy to get that $\alpha_{k}(t)$ is the lower solution of (1.1) and $\alpha_{k+1}(t)$ satisfies

$$
\left\{\begin{aligned}
\alpha_{k+1}(t) & =\alpha_{k+1}(0) t^{1-q} \\
& +\frac{1}{\Gamma(q)} \int_{0}^{t}(t-s)^{q-1}\left[f\left(s, \alpha_{k}(s)\right)+f_{x}\left(s, \alpha_{k}(s)\right)\left(\alpha_{k+1}(s)-\alpha_{k}(s)\right)\right] d s, \\
\tilde{\alpha_{k+1}}(0) & =g\left(\tilde{\alpha_{k}}(T)\right)+g^{\prime}\left(\tilde{\alpha_{k}}(T)\right)\left(\alpha_{k+1}(T)-\tilde{\alpha_{k}}(T)\right) .
\end{aligned}\right.
$$

So using the above method we can obtain $t^{1-q} \alpha_{k}(t) \leq t^{1-q} \alpha_{k+1}(t)$.

Similarly, $\beta_{k}(t)$ is the upper solution of (1.1) and $\beta_{k+1}(t)$ satisfies

$$
\left\{\begin{aligned}
\beta_{k+1}(t) & =\tilde{\beta_{k+1}}(0) t^{q-1} \\
& +\frac{1}{\Gamma(q)} \int_{0}^{t}(t-s)^{q-1}\left[f\left(s, \beta_{k}(s)\right)+f_{x}\left(s, \alpha_{k}(s)\right)\left(\beta_{k+1}(s)-\beta_{k}(s)\right)\right] d s, \\
\tilde{\beta_{k+1}}(0) & =g\left(\tilde{\beta_{k}}(T)\right)+g^{\prime}\left(\tilde{\alpha_{k}}(T)\right)\left(\tilde{\beta}_{k+1}(T)-\tilde{\beta_{k}}(T)\right) .
\end{aligned}\right.
$$

Hence $t^{1-q} \beta_{k+1}(t) \leq t^{1-q} \beta_{k}(t)$.

To show $t^{1-q} \alpha_{k+1}(t) \leq t^{1-q} \beta_{k+1}(t)$, we set $p(t)=\alpha_{k+1}(t)-\beta_{k+1}(t)$.

$$
\begin{gathered}
p(t)=\alpha_{k+1}(t)-\beta_{k+1}(t) \\
=\alpha_{k+1}^{\tilde{k}}(0) t^{q-1} \\
+\frac{1}{\Gamma(q)} \int_{0}^{t}(t-s)^{q-1}\left[f\left(s, \alpha_{k}(s)\right)+f_{x}\left(s, \alpha_{k}(s)\right)\left(\alpha_{k+1}(s)-\alpha_{k}(s)\right) d s\right] \\
-\beta_{k+1}(0) t^{q-1} \\
-\frac{1}{\Gamma(q)} \int_{0}^{t}(t-s)^{q-1}\left[f\left(s, \beta_{k}(s)\right)+f_{x}\left(s, \alpha_{k}(s)\right)\left(\beta_{k+1}(s)-\beta_{k}(s)\right)\right] d s \\
\leq \tilde{p}(0) t^{q-1}+\frac{1}{\Gamma(q)} \int_{0}^{t}(t-s)^{q-1} f_{x}\left(s, \alpha_{k}(s)\right) p(s) d s .
\end{gathered}
$$

Then we have that

$$
\begin{aligned}
\tilde{p}(0) & =\tilde{\alpha_{k+1}}(0)-\tilde{\beta_{k+1}}(0) \\
& =g\left(\tilde{\alpha_{k}}(T)\right)-g\left(\tilde{\beta_{k}}(T)\right)+g^{\prime}\left(\tilde{\alpha_{k}}(T)\right)\left[\tilde{\alpha_{k+1}}(T)-\tilde{\alpha_{k}}(T)-\tilde{\beta_{k+1}}(T)+\tilde{\beta_{k}}(T)\right] \\
& \leq g^{\prime}\left(\tilde{\alpha_{k}}(T)\right) \tilde{p}(T) .
\end{aligned}
$$

By Lemma 4, we know $t^{1-q} \alpha_{k+1}(t) \leq t^{1-q} \beta_{k+1}(t)$.

By induction, we easily get $\left\{t^{1-q} \alpha_{n}\right\},\left\{t^{1-q} \beta_{n}\right\}$ which satisfy the relation

$$
t^{1-q} \alpha_{0} \leq t^{1-q} \alpha_{1} \leq \cdots \leq t^{1-q} \alpha_{n} \leq \cdots \leq t^{1-q} \beta_{n} \leq \cdots \leq t^{1-q} \beta_{1} \leq t^{1-q} \beta_{0} .
$$

Obviously, the sequences $\left\{t^{1-q} \alpha_{n}\right\},\left\{t^{1-q} \beta_{n}\right\}$ are uniformly bounded and equicontinuous. Hence by the Ascoli-Arzela Theorem that the sequences $\left\{t^{1-q} \alpha_{n}\right\},\left\{t^{1-q} \beta_{n}\right\}$ converge uniformly on $J$ with

$$
\lim _{n \rightarrow \infty} t^{1-q} \alpha_{n}=t^{1-q} \alpha, \lim _{n \rightarrow \infty} t^{1-q} \beta_{n}=t^{1-q} \beta .
$$


Then we can easily show that $\alpha$ and $\beta$ are the solutions of (1.1) in $\left[\alpha_{0}, \beta_{0}\right]$.

To prove quadratic convergence of $\left\{t^{1-q} \alpha_{n}\right\},\left\{t^{1-q} \beta_{n}\right\}$ to the solution, we consider

$$
p_{k+1}=x-\alpha_{k+1}, \quad r_{k+1}=\beta_{k+1}-x .
$$

We can obtain

$$
\begin{aligned}
\tilde{p_{k+1}}(0) & =\tilde{x}(0)-\tilde{\alpha_{k+1}}(0) \\
& =g(\tilde{x}(T))-g\left(\tilde{\alpha_{k}}(T)\right)-g^{\prime}\left(\tilde{\alpha_{k}}(T)\right)\left(\tilde{\alpha_{k+1}}(T)-\tilde{\alpha_{k}}(T)\right) \\
& =g^{\prime}(\tilde{\theta}(T)) \tilde{p_{k}}(T)-g^{\prime}\left(\tilde{\alpha_{k}}(T)\right)\left(\tilde{p_{k}}(T)-p_{\hat{k+1}}(T)\right) \\
& \leq L_{1}\left|\tilde{\theta}(T)-\tilde{\alpha_{k}}(T)\right| \tilde{p_{k}}(T)+g^{\prime}\left(\tilde{\alpha_{k}}(T)\right) p_{\tilde{k+1}}(T) \\
& \leq L_{1}\left\|p_{k}(t)\right\|_{C_{1-q}}^{2}+N\left\|p_{k+1}(t)\right\|_{C_{1-q}} .
\end{aligned}
$$

Then we have that

$$
\begin{aligned}
& p_{k+1}(t)=x(t)-\alpha_{k+1}(t) \\
& =p_{k+1}(0) t^{q-1}+\frac{1}{\Gamma(q)} \int_{0}^{t}(t-s)^{q-1}\left[f(s, x(s))-f\left(s, \alpha_{k}(s)\right)\right. \\
& \left.-f_{x}\left(s, \alpha_{k}(s)\right)\left(\alpha_{k+1}(s)-\alpha_{k}(s)\right)\right] d s \\
& =p_{k+1}(0) t^{q-1}+\frac{1}{\Gamma(q)} \int_{0}^{t}(t-s)^{q-1}\left[f_{x}(s, \rho(s)) p_{k}(s)-f_{x}\left(s, \alpha_{k}(s)\right) p_{k}(s)\right. \\
& \left.\left.+f_{x}\left(s, \alpha_{k}(s)\right) p_{k+1}(s)\right)\right] d s \\
& \leq p_{k+1}(0) t^{q-1}+\frac{1}{\Gamma(q)} \int_{0}^{t}(t-s)^{q-1}\left[L_{2}\left(\rho(s)-\alpha_{k}(s)\right) p_{k}(s)+M p_{k+1}(s)\right] d s \\
& \leq p_{k+1}(0) t^{q-1}+\frac{1}{\Gamma(q)} \int_{0}^{t}(t-s)^{q-1}\left[L_{2}\left(x(s)-\alpha_{k}(s)\right) p_{k}(s)+M p_{k+1}(s)\right] d s \\
& \leq p_{k+1}(0) t^{q-1}+\frac{1}{\Gamma(q)} \int_{0}^{t}(t-s)^{q-1}\left(L_{2} s^{2 q-2}\left\|p_{k}(t)\right\|_{C_{1-q}}^{2}+M p_{k+1}(s)\right) d s .
\end{aligned}
$$

Then using the method of successive approximations we get that

$$
\begin{gathered}
p_{k+1}(t) \\
\leq \Gamma(q) \tilde{p_{k+1}}(0) t^{q-1} E_{q, q}\left(M t^{q}\right) \\
+L_{2}\left\|p_{k}(t)\right\|_{C_{1-q}}^{2} \int_{0}^{t}(t-s)^{q-1} s^{2 q-2} E_{q, q}\left(M(t-s)^{q}\right) d s \\
\leq \Gamma(q) E_{q, q}\left(M T^{q}\right) p_{\tilde{k}+1}(0) t^{q-1} \\
+L_{2}\left\|p_{k}(t)\right\|_{C_{1-q}}^{2} E_{q, q}\left(M(T)^{q}\right) \frac{\Gamma(q) \Gamma(2 q-1)}{\Gamma(3 q-1)} t^{3 q-2} . \\
t^{1-q} p_{k+1}(t) \leq \Gamma(q) p_{\tilde{k+1}}(0) E_{q, q}\left(M T^{q}\right)
\end{gathered}
$$




$$
\begin{gathered}
+L_{2}\left\|p_{k}(t)\right\|_{C_{1-q}}^{2} E_{q, q}\left(M(T)^{q}\right) \frac{\Gamma(q) \Gamma(2 q-1)}{\Gamma(3 q-1)} T^{2 q-1} \\
\leq\left(L_{1}\left\|p_{k}(t)\right\|_{C_{1-q}}^{2}+N\left\|p_{k+1}(t)\right\|{C_{1-q}}_{1-}\right) \Gamma(q) E_{q, q}\left(M T^{q}\right) \\
+L_{2}\left\|p_{k}(t)\right\|_{C_{1-q}}^{2} E_{q, q}\left(M(T)^{q}\right) \frac{\Gamma(q) \Gamma(2 q-1)}{\Gamma(3 q-1)} T^{2 q-1} \\
=\left(L_{1} \Gamma(q)+L_{2} \frac{\Gamma(q) \Gamma(2 q-1)}{\Gamma(3 q-1)} T^{2 q-1}\right) E_{q, q}\left(M T^{q}\right)\left\|p_{k}(t)\right\|_{C_{1-q}}^{2} \\
+\Gamma(q) E_{q, q}\left(M T^{q}\right) N\left\|p_{k+1}(t)\right\|_{C_{1-q}},
\end{gathered}
$$

where $E_{q}(t)=\sum_{k=0}^{\infty} \frac{t^{k}}{\Gamma(q k+1)}, \quad E_{q, q}(t)=\sum_{k=0}^{\infty} \frac{t^{k}}{\Gamma(q k+q)}$.

Thus we have the estimate

$$
\left\|p_{k+1}(t)\right\|_{C_{1-q}} \leq \Omega\left\|p_{k}(t)\right\|_{C_{1-q}}^{2}
$$

where

$$
\Omega=\frac{\left(L_{1} \Gamma(q)+L_{2} \frac{\Gamma(q) \Gamma(2 q-1)}{\Gamma(3 q-1)} T^{2 q-1}\right) E_{q, q}\left(M T^{q}\right)}{1-\Gamma(q) E_{q, q}\left(M T^{q}\right) N} .
$$

Similarly

$$
\left\|r_{k+1}(t)\right\|_{C_{1-q}} \leq \Omega\left\|r_{k}(t)\right\|_{C_{1-q}}^{2}
$$

\section{REFERENCES}

[1] M. Benchohra, S. Hamani, and S. K. Ntouyas, "Boundary value problems for differential equations with fractional order and nonlocal conditions," Nonlinear Anal., vol. 71, pp. 2391-2396, 2009.

[2] J. V. Devi, F. A. McRae, and Z. Drici, "Generalized quasilinearization for fractional differential equations," Comput. Math. Appl., vol. 59, pp. 1057-1062, 2010.

[3] J. V. Devi and V. Radhika, "Quasilinearization for hybrid caputo fractional differential equations," Dynamic Systems and Applications, vol. 21, pp. 567-582, 2012.

[4] J. V. Devi and C. Suseela, "Quasilinearization for fractional differential equations," Commun. Appl. Anal., vol. 12, pp. 407-418, 2008.

[5] A. M. A. El-sayed and H. H. G. Hashem, "Existence results for nonlinear quadratic functional integral equations of fractional orders," Miskolc Math. Notes, vol. 14, pp. 79-88, 2013.

[6] R. W. Ibrahim, "Global controllability of a set of fractional differential equations," Miskolc Math. Notes, vol. 12, pp. 51-60, 2011.

[7] A. A. Kilbas, S. G. Samko, and O. I. Marichev, Fractional Integral and Derivatives,Theory and Applications. Gordon and Breach, Yverdon, 1993.

[8] A. A. Kilbas, H. M. Srivastava, and J. J. Trujillo, Theory and Applications of Fractional Differential Equations. Elservier Science B.V., Amsterdam, 2006.

[9] V. Lakshmikanthan, S. Leela, and J. V. Devi, Theory of Fractional Dynamic Systems. Cambridge Academic Publishers, Cambridge, 2009.

[10] V. Lakshmikanthan and A. S. Vatsala, Generalized quasilinearization for nonlinear problems. Academic Publications,Dordrechel, 1998. 
[11] X. Liu, Z. Liu, and X. Fu, "Relaxation in nonconvex optimal control problems described by fractional differential equations," Journal of Mathematical Analysis and Applications, vol. 409, pp. 446-458, 2014.

[12] Z. H. Liu, X. W. Li, and J. H. Sun, "Controllability of nonlinear fractional impulsive evolution systems," Journal of Integral Equations and Applications, vol. 25(3), pp. 395-405, 2013.

[13] Z. H. Liu and J. H. Sun, "Nonlinear boundary value problems of fractional functional integrodifferential equations," Computers and Mathematics with Applications, vol. 64, pp. 3228-3234, 2012.

[14] Z. H. Liu, J. H. Sun, and I. Szanto, "Monotone iterative technique for riemann-liouville fractional integro-differential equations with advaced arguments," Results in Math., vol. 63, pp. 1277-1287, 2013.

[15] Z. Liu and X. Li, "On the controllability of impulsive fractional evolution inclusions in banach spaces," J Optim Theory Appl, vol. 156, pp. 167-182, 2013.

[16] I. Podlubny, Fractional Differential Equations. Academic Press, San Diego, 1999.

[17] A. Rontó and M. Rontó, "Periodic successive approximations and interval halving," Miskolc Math. Notes, vol. 13, pp. 459-482, 2012.

[18] A. Rontó and M. Rontó, "On constructive investigation of a class of nonlinear boundary value problems for functional differential equations," Carpathian J. Math., vol. 29, pp. 91-108, 2013.

[19] M. Rontó and K. Marynets, "On the parametrization of boundary-value problems with three-point non-linear restrictions," Miskolc Math. Notes, vol. 13, pp. 91-106, 2012.

[20] J. Sabatier, O. P. Agrawal, and J. A. T. Machado, Advances in Fractional Calculus: Theoretical Developments and Applications in Physics and Engineering. Springer, Dordrecht, 2007.

[21] G. Wang, R. P. Agarwal, and A. Cabada, "Existence results and the monotone iterative technique for systems of nonlinear fractional differential equations," Appl. Math. Lett., vol. 25, pp. 10191024, 2012.

[22] J. R. Wang, W. Wei, and M. Fečkan, "Nonlocal cauchy problems for fractional evolution equations involving volterra-fredholm type integral operators," Miskolc Math. Notes, vol. 13, pp. 127-147, 2012.

[23] W. Y. Zhong and W. Lin, "Nonlocal and multiple-point boundary value problem for fractional differential equations," Comput. Math. Appl., vol. 59, pp. 1345-1351, 2010.

Authors' addresses

Zhenhai Liu

Guangxi Key Laboratory of Hybrid Computation and IC Design Analysis and College of Sciences, Guangxi University for Nationalities, Nanning, Guangxi 530006, P.R. China

E-mail address: zhhliu@hotmail.com

\section{Rui Wang}

College of Sciences, Guangxi University for Nationalities, Nanning, Guangxi 530006, P.R. China

E-mail address: $278349201 @ q q . c o m$

Jing Zhao

College of Sciences, Guangxi University for Nationalities, Nanning, Guangxi 530006, P.R. China

E-mail address: jingzhao1000126.com 